\title{
The impact of wearable cardioverter-defibrillator use on long-term decision for implantation of a cardioverter-defibrillator in a semirural acute care hospital
}

\author{
Anil-Martin Sinha ${ }^{1,2} \cdot$ Jens Bense ${ }^{1,2} \cdot$ Wolfgang Hohenforst-Schmidt ${ }^{1,2}$ \\ Received: 2 August 2020 / Accepted: 12 October 2020 / Published online: 17 November 2020 \\ (C) The Author(s) 2020
}

\begin{abstract}
Purpose Large-scale multi-center studies have reported on efficacy of the wearable cardioverter-defibrillator (WCD). However, outcomes focused on WCD patients treated at community-based acute care centers are lacking.

Methods Patients with cardiomyopathy were included when left ventricular ejection fraction (LVEF) at baseline was $\leq 35 \%$. There were 120 patients meeting the criteria who also had LVEF measured at baseline and after 90 days of WCD use.

Results After 90 days of WCD use, there were 44 (37\%) patients in whom LVEF improved to $>35 \%$. Comparison of patients, by whether LVEF improved or not, indicated that median days of WCD wear and hours of daily use were similar as well as characteristics, such as gender, age, and starting LVEF; and diagnoses leading to WCD prescription were similar between groups as were symptom-based prescription of medications. At the end of WCD use, improved LVEF $>35 \%$ correlated with fewer implantable cardioverter-defibrillator (ICD) implants. There were 4 (3\%) episodes of new atrial fibrillation detected during WCD use. The WCD appropriately delivered a shock to 3 (2.5\%) patients with VT/VF being terminated by the first shock. All shocked patients survived for at least $24 \mathrm{~h}$ post-shock.

Conclusions During WCD use, ischemic and non-ischemic cardiomyopathy patients manifest improved LVEF by 90 days. Long-term care decisions, such as implantation of an ICD, were influenced by LVEF improvement and occurrence of spontaneous VT/VF. The WCD protected patients from sudden cardiac death $(\mathrm{SCD})$ until patient response to guideline-directed medical therapy could be determined.
\end{abstract}

Keywords Arrhythmia · Cardiomyopathy · Implantable cardioverter-defibrillator · Sudden cardiac arrest · Sudden cardiac death · Wearable cardioverter-defibrillator

\section{Abbreviations}

DCM Dilative cardiomyopathy

ECG Electrocardiogram

ESC European Society of Cardiology

EMS Emergency medical services

GDMT Guideline-directed medical therapy

ICD Implantable cardioverter-defibrillator

ICM Ischemic cardiomyopathy

IQR Interquartile range
LVEF Left ventricular ejection fraction

MI Myocardial infarction

NICM Non-ischemic cardiomyopathy

PCI Percutaneous coronary intervention

SCD Sudden cardiac death

VEST Vest Prevention of Early Sudden Death Trial

VF Ventricular fibrillation

VT Ventricular tachycardia

WCD Wearable cardioverter-defibrillator
Anil-Martin Sinha anil-martin.sinha@sana.de

1 Department of Cardiology, Sana Klinikum Hof, Eppenreuther Str. 9, 95032 Hof, Germany

2 Department of Cardiology, Teaching Hospital of the Friedrich Alexander University Erlangen/Nürnberg, Erlangen/ Nürnberg, Germany

\section{Introduction}

Outside the hospital, acute care is dependent on access to emergency medical services (EMS). Patients who reside in semirural settings have inherently longer EMS response times $[1,2]$. Following onset of ventricular tachycardia (VT) or ventricular fibrillation (VF), the likelihood of successful 
resuscitation decreases at a rate of approximately $10 \%$ per minute of delayed defibrillation [3]. However, automated defibrillators placed in the home have been unsuccessful when measured against improved survival following VT/VF [4].

Although the Vest Prevention of Early Sudden Death Trial (VEST) failed to achieve statistical significance for sudden cardiac death (SCD) reduction at 90 days [5], multi-center registries and prospective studies have provided convincing evidence that patients benefit from wearable cardioverterdefibrillator (WCD) use [6, 7]. Feldman et al. found in 289 patients at high risk for sudden death that a WCD was beneficial in detecting and effectively treating ventricular tachyarrhythmias in patients at high risk for sudden death who were not clear candidates for an implantable cardioverterdefibrillator (ICD) [8]. Moreover, the WCD seemed to be useful as a bridge to transplantation or ICD in selected patients [8].

As the risk for sudden cardiac arrest during the waiting period before ICD implantation was unclear, Epstein et al. analyzed detected arrhythmias and shocks delivered in 853 WCD patients with reduced left ventricular ejection fraction (LVEF) during the first 3 months post-myocardial infarction (MI) [9]. They found that $1.4 \%$ of patients were successfully shocked within the first 3 months with a resuscitation survival rate of $91 \%$. Furthermore, sudden cardiac death risk in postMI patients was highest in the first month of WCD use [9]. Chung et al. assessed patient compliance and effectiveness of antiarrhythmic treatment by WCD in 3569 patients [10]. They reported that compliance was satisfactory with $90 \%$ wear time in $50 \%$ of patients and low sudden death mortality during use, and that survival was comparable to that of ICD patients. [10].

However, there are few reports about WCD experience when patients are discharged from community-based hospitals. The current study prospectively enrolled patients with increased risk of VT/VF indicated by cardiomyopathy and impaired LVEF, and presents the WCD experience from a single-center acute care hospital serving a semirural region.

\section{Methods}

\subsection{Consent}

Before the start of WCD wear, all patients are consented to use their data for quality monitoring, healthcare operations, and research purposes.

\subsection{Cohort}

The prospective analysis included patients prescribed the WCD at the Sana Klinikum Hof, Germany, from February 2012 to May 2017. Prescription criteria were based upon clinical characteristics at hospital admission which were LVEF $\leq 35 \%$ and either ischemic $(n=46,38 \%$; acute myocardial infarction or ischemic cardiomyopathy), non-ischemic ( $n=69,58 \%$; myocarditis, congestive heart failure, or dilated cardiomyopathy), or others $(n=5,3 \%$; ICD infection leading to explant or genetic cardiomyopathy). Patients were enrolled if they were prescribed the WCD, presented with LVEF $\leq 35 \%$ and cardiomyopathy, and had LVEF measured after 90 days of WCD use. LVEF was measured using Simpson's biplane method.

\subsection{Patient characteristics}

Patient characteristics and medical treatment were evaluated at the beginning and after 90 days of WCD use.

\subsection{Wearable cardioverter-defibrillator use}

Patient use data were obtained from the commercial database. Days of wear was the sum of days in which the WCD was worn for greater than $15 \mathrm{~min}$. Hours of daily use was the ratio of the sum of hours and the sum of days minus 1 . The purpose of the 1-day adjustment was to correct for the partial days available on the first and last days of prescribed wear.

\subsection{Arrhythmia data analysis}

Electrocardiogram (ECG) data recorded by the WCD system were remotely transmitted, screened for whether they included information on heart rate and rhythm, and analyzed by the authors, including 2 cardiologists. The WCD recorded the automated detection of and delivered shock in response to VT and VF events. The WCD includes a 2-lead ECG monitoring system and continuously monitors the patient for heart rate and rhythm. In the event of VT or VF, the WCD will record the ECG immediately before and after delivering a shock. Signals other than VT/ VF can also initiate an ECG recording. These include irregular and fast heart rhythms that are of supraventricular origin. The lengths of recording typically vary between $45 \mathrm{~s}$ and several minutes depending of the nature of the detected heart rhythm and the patient's interaction with the device's response buttons. VT or VF was ventricular tachyarrhythmia lasting longer than $30 \mathrm{~s}$. VT was defined as having monomorphic or polymorphic characteristics, and VF as an inconsistent morphology. A single event included all ECG records of VT or VF occurring within $24 \mathrm{~h}$ of the index arrhythmia. Appropriate shocks were defined as being delivered in response to an episode of VT or VF. Inappropriate shocks were WCD shocks delivered in the absence of VT or VF.

\subsection{Post-shock follow-up}

Data associated with shock events include the ECG record encompassing the event, the number of shocks administered, and whether shock resulted in conversion. All shock events were 
investigated by the responsible physician. Outcomes were tabulated, with survival defined as alive $24 \mathrm{~h}$ after receiving a shock.

\subsection{Statistical analysis}

Statistical analysis was performed using R [11]. Data were reported as the median and interquartile range (IQR) or as the number of patients and percentage of total patients. Categorical data were analyzed using Fisher's exact test and continuous data by the Wilcoxon test.

\section{Results}

\subsection{Patient selection and characteristics}

WCD prescription was based upon standard clinical algorithm performed at the Sana Klinikum in Hof, Germany (Fig. 1). Eligible patients diagnosed with acute MI were prescribed the WCD for 3 months when percutaneous coronary intervention (PCI) was performed or for 40 days without revascularization. Prescription of the WCD was for 3 months when the diagnosis was non-ischemic, non-acute ischemic, or other eligible cardiomyopathy. At the end of WCD prescription, LVEF was reevaluated and decision to implant an ICD followed European Society of Cardiology (ESC) guidelines.

Baseline patient characteristics are summarized in Table 1. The 120 patients were primarily male (79\%) with median age 66 years. Cardiomyopathy leading to WCD prescription were primarily ischemic and non-ischemic (38\% versus $58 \%$ ) with reduced LVEF $\leq 35 \%$. All patients received optimized medication for heart failure, including beta blocker (92\%), ACE inhibitor (80\%), and diuretic (86\%).

\subsection{Patient characteristics after 90 days}

Patient characteristics after 90 days of WCD use, distributed by whether LVEF surpassed $35 \%$ or not, are also summarized in Table 1. The distribution of gender ( $80 \%$ versus $77 \%$ male), median age (69 versus 64 years), and starting LVEF (26\% versus $26 \%$ ) was similar between both groups. The percentage of patients who achieved LVEF $>35 \%$ at 90 days was also similar for ischemic, non-ischemic, and other cardiomyopathy diagnoses. Symptom-based prescription of medication (e.g., beta-blockers, ACE inhibitors, diuretics and digitalis) was similar when compared by LVEF at follow-up. Comparison of WCD use by patients in these two groups indicated that median days of wear (52 versus 45 days) and hours of daily use (23.0 versus $22.7 \mathrm{~h}$, an $18 \mathrm{~min}$ difference) were also similar. Overall, the WCD was worn by all patients for at least 3 days, and 112 (93\%), 94 (78\%), 33 (51\%), and 11 (9\%) patients wore the WCD for at least 7, 30, 60, and 90 days, respectively. Other than WCD delivered shock preceding an ICD implant, reasons for discontinuing WCD use prior to 90 days were not recorded during the study period.

\subsection{LVEF after 90 days follow-up}

At the start of WCD wear, all patients had LVEF $\leq 35 \%$ with LVEF 25 to $30 \%$ being the most frequent, accounting
Fig. 1 Sana Klinikum Hof, Germany, algorithm for prescribing the wearable cardioverter-defibrillator (MI, myocardial infarction; DCM/ ICM, dilative cardiomyopathy/ ischemic cardiomyopathy; ICD, implantable cardioverterdefibrillator; ACE-I, angiotensin converting enzyme inhibitor)

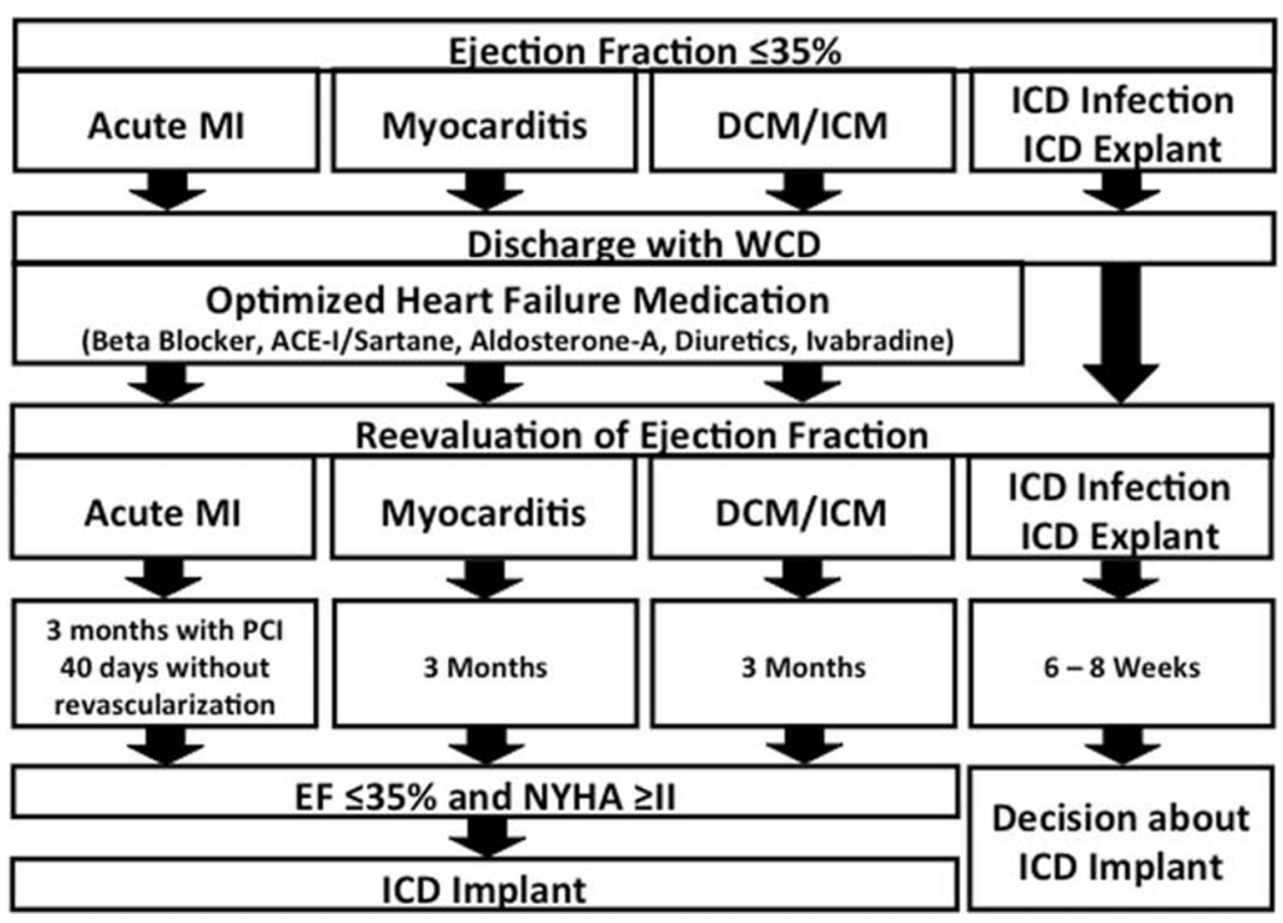


Table 1 Wearable cardioverterdefibrillator patient characteristics

\begin{tabular}{|c|c|c|c|c|}
\hline Characteristic & Included, $N=120$ & $\begin{array}{l}90 \text { day LVEF } \\
\leq 35 \%, N=76\end{array}$ & $\begin{array}{l}90 \text { day LVEF } \\
>35 \%, N=44\end{array}$ & $p$ value \\
\hline \multicolumn{5}{|l|}{ Demographics } \\
\hline Male, $N(\%)$ & $95(79)$ & $61(80)$ & $34(77)$ & 0.82 \\
\hline Age, median (IQR) & $66(56,75)$ & $69(54,74)$ & $64(54,74)$ & 0.11 \\
\hline LVEF at start, median (IQR) & $26(20,30)$ & $26(22,30)$ & $26(22,30)$ & 0.98 \\
\hline \multicolumn{5}{|l|}{ Reason for WCD } \\
\hline Ischemic, $N(\%)$ & $46(38)$ & $29(38)$ & $17(39)$ & 1 \\
\hline Non-Ischemic, $N(\%)$ & $69(58)$ & $44(58)$ & $25(57)$ & 1 \\
\hline Others, $N(\%)$ & $5(4)$ & $3(4)$ & $2(5)$ & 1 \\
\hline \multicolumn{5}{|l|}{ Medications } \\
\hline Beta blocker, $N(\%)$ & $111(92)$ & $70(92)$ & $41(93)$ & 1 \\
\hline ACE inhibitor, $N(\%)$ & $96(80)$ & $61(80)$ & $35(80)$ & 1 \\
\hline Diuretic, $N(\%)$ & $103(86)$ & $64(84)$ & $39(89)$ & 0.59 \\
\hline Digitalis, $N(\%)$ & $2(2)$ & $1(1)$ & $1(2)$ & 1 \\
\hline \multicolumn{5}{|l|}{ WCD use } \\
\hline Days of wear, median (IQR) & $48(37,62)$ & $52(40,66)$ & $45(36,56)$ & 0.14 \\
\hline Hours daily use, median (IQR) & $22.9(21.2,23.4)$ & $23.0(21.4,23.4)$ & $22.7(20.1,23.3)$ & 0.30 \\
\hline \multicolumn{5}{|l|}{ ICD implanted at 90 days } \\
\hline Received ICD, $N(\%)$ & $71(59)$ & $68(89)$ & $3(7)$ & $<0.001$ \\
\hline No ICD, $N(\%)$ & $44(37)$ & $3(4)$ & 41 (93) & $<0.001$ \\
\hline Unknown, $N(\%)$ & $5(4)$ & $5(7)$ & $0(0)$ & 0.16 \\
\hline
\end{tabular}

$I C D$, implantable cardioverter-defibrillator; $I Q R$, interquartile range; $N$, number; $W C D$, wearable cardioverterdefibrillator for $49(41 \%)$ patients (Fig. 2, left panel). After 90 days of WCD wear, there were $76(63 \%)$ patients with LVEF $\leq$ $35 \%$ remaining while $44(37 \%)$ responded to guidelinedirected medical therapy (GDMT) as indicated by improved LVEF $>35 \%$ (Fig. 2, right panel). Analysis of patients by starting LVEF indicated that patients with severely reduced $\mathrm{LVEF} \leq 25 \%$ were as likely to improve as were patients with LVEF above $25 \%$. Thus, after 90 days of WCD wear, 22 out of 58 patients with LVEF $\leq 25 \%$ improved to LVEF $>35 \%$, while 22 out of 62 patients with LVEF $>25 \%$ improved to $\mathrm{LVEF}>35 \%$, equivalent to one-third of patients.
Fig. 2 Histogram of patient left ventricular ejection fraction (LVEF) before (left panel) and after 90 days of wearable cardioverter-defibrillator use (right panel)

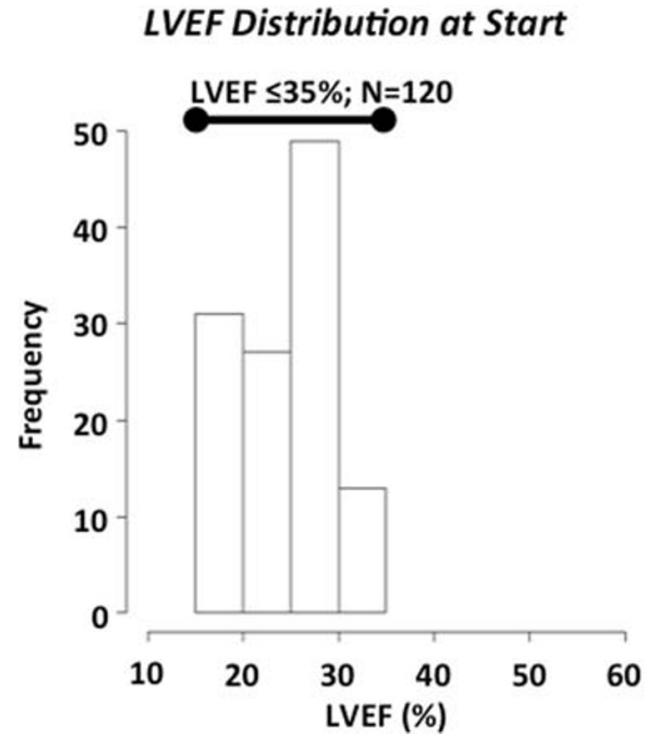




\subsection{Appropriate wearable cardioverter-defibrillator therapy}

Three patients (2.5\%) ages 60, 78, and 67 years were shocked for VT/VF, 1 non-ischemic, and 2 ischemic (Table 2). In each patient, a single episode of VT/VF was detected on days of WCD wear 36, 6, and 17, and terminated by the first shock. No patient was shocked more than once and no patient was shocked inappropriately by the WCD or died while wearing the WCD. Survival was documented at least $24 \mathrm{~h}$ post-shock. The shocked patients were successfully implanted with an ICD for secondary prevention of SCD.

\subsection{Remote monitoring arrhythmia detection}

During our study, WCD-based remote monitoring of 2794 ECG recordings were available. Arrhythmia monitoring beyond occurrence of VT/VF was reported via automatic downloads of ECG records when non-sinus rhythm was detected. During the 90 days of prescribed wear, new atrial fibrillation was detected in $1(1 \%) \mathrm{LVEF} \leq 35 \%$ and $3(7 \%) \mathrm{LVEF}>35 \%$ patients. A total of 2202 recordings in 96 patients $(80 \%)$ were artifacts (non-VT/VF, non-atrial fibrillation). In total, 60 patients $(50 \%)$ initiated 443 recordings manually by pressing the WCD response buttons for $3 \mathrm{~s}$. There was no other supraventricular arrhythmia detected.

\subsection{ICD implantation}

In the absence of VT/VF, the decision to implant an ICD correlated with LVEF at follow-up (Table 1). For example, $68(89 \%)$ patients in the LVEF < 35\% group versus $3(7 \%)$ among the LVEF $>35 \%$ group were implanted with an ICD

Table 2 Wearable cardioverter-defibrillator shocks

\begin{tabular}{|c|c|c|c|}
\hline & Patient 1 & Patient 2 & Patient 3 \\
\hline \multicolumn{4}{|l|}{ Demographics } \\
\hline Gender & Female & Male & Male \\
\hline Age & 60 & 78 & 67 \\
\hline LVEF at start & $30 \%$ & $20 \%$ & $20 \%$ \\
\hline Reason for WCD & Non-ischemic & Ischemic & Ischemic \\
\hline \multicolumn{4}{|l|}{ Shocks } \\
\hline Day of Shock & 36 & 6 & 17 \\
\hline Pre-shock rhythm & VT & $\mathrm{VF}$ & $\mathrm{VF}$ \\
\hline Number of shocks & 1 & 1 & 1 \\
\hline Survival at $24 \mathrm{~h}$ & Yes & Yes & Yes \\
\hline \multicolumn{4}{|l|}{ ICD implanted } \\
\hline Received ICD & Yes & Yes & Yes \\
\hline
\end{tabular}

$I C D$, implantable cardioverter-defibrillator; $V F$, ventricular fibrillation; $V T$, ventricular tachycardia; $W C D$, wearable cardioverter-defibrillator for prevention of SCD $(p<0.001)$. There were, however, 5 (4\%) patients in the LVEF $\leq 35 \%$ group, where the decision to implant was unavailable and corresponded with patient decision to receive follow-up care at another institution.

Subgroup analysis of the reason for WCD prescription along with the decision whether to implant an ICD after 90 days of WCD use indicated that among the LVEF $<35 \%$ group, there were 29 out of 29 ICM and 39 out of 44 NICM patients implanted with an ICD. In contrast, among the LVEF $>35 \%$ group, there were 15 out of 17 ICM patients and 24 out of 25 NICM patients who were not implanted.

\section{Discussion}

The goal of our analysis was to evaluate whether patients residing in a semirural region and cared for at a community hospital benefitted from wearing the WCD during optimization of GDMT. Our single-center experience with a semirural patient population compared favorably to previously reported outcomes of WCD patients in Germany. The PROLONG cohort reported outcomes from 156 patients enrolled at a single center located in urban Hannover, Germany [12]. They identified LVEF improvement during 90 days of WCD use of 58 (37\%) and reported VT/VF events in $11(7 \%)$ patients [12]. These results were similar to the outcomes from the community acute care hospital, in which $44(37 \%)$ patients improved LVEF to $>35 \%$ after 90 days and recorded $3(2.5 \%)$ VT/VF events. The PROLONG investigators reported also on a subanalysis of 117 non-ischemic cardiomyopathy (NICM) patients and identified $6(5 \%)$ in whom VT/VF occurred during WCD use [13].

In an independent publication, the German national experience with the WCD reported that out of 6043 patients, 1.3\% of non-ischemic and $1.4 \%$ of ischemic patients received an appropriate shock for VT/VF [6]. Similarly, when WCD use among the semirural patient population was examined, VT/ VF also occurred among both categories, 1 out of 69 nonischemic and 2 out of 46 ischemic patients. In the current study, the 3 patients shocked for VT/VF were converted by the first shock; these patients survived long enough to receive an ICD implant and no patient in the current study received an inappropriate shock, not unexpectedly given the $0.4 \%$ frequency of inappropriate shocks reported previously in Germany [6]. Our investigation confirmed that improved LVEF $>35 \%$ can be achieved by a large number of patients, and agree with the German national experience and the PROLONG study that newly diagnosed cardiomyopathy patients, including those with NICM and LVEF $\leq 35 \%$, were at elevated risk of VT/VF during the period of LVEF recovery $[6,12,13]$.

In clinical practice, the recovery process and titration of medication under GDMT cannot be hurried. Use of the 
WCD allows time to become part of the therapy prior to risk stratification for permanent SCD prevention in patients diagnosed with ischemic as well as non-ischemic cardiomyopathy. After 90 days of GDMT with WCD use, 41 (93\%) of the 44 patients with improved LVEF $>35 \%$ did not receive an ICD. In contrast, 68 (89\%) of the 76 patients with LVEF $\leq 35 \%$ at 90 days were ICD implanted. Moreover, one-third of patients improved their LVEF to $>35 \%$ independent of the severity of LVEF impairment, age, or gender. Sub-analysis of the semirural patients by starting LVEF $\leq 25 \%$ or $>25 \%$ indicated that after 90 days of WCD wear, one-third of patients from either group responded to GDMT with improved LVEF $>35 \%$. This illustrates the difficulty of separating patients who will recover from those who will not prior to optimization of GDMT.

Evidence that the WCD patient benefits in ways beyond delivery of shock were also provided by the WEARIT-II study, a prospective registry of $2000 \mathrm{WCD}$ patients from the USA [7]. In addition to detection of VT/VF in 41 (2.1\%) patients, the WEARIT-II investigators reported that the WCD recorded non-sustained VT in $28(1.4 \%)$ patients and new atrial arrhythmia in $72(3.6 \%)$. Consistent with WEARIT-II, the current study identified 4 (3.3\%) patients with new atrial fibrillation.

In contrast to results reported by observational studies $[6-10,12,13]$, the interventional randomized control VEST failed to achieve statistical significance for the primary endpoint of SCD reduction at 90 days [5]. However, interpretation of the primary endpoint is confounded by the likelihood that the study was underpowered due to less than anticipated daily wear of the WCD and the potential to undercount arrhythmic death $[5,14]$. When considered along with observational studies, the results of VEST demand a nuanced approach when discussing use of the WCD with the at risk patient.

Although exploratory, as-treated analyses of VEST identified significantly fewer deaths among patients wearing the WCD while indicating that the majority of arrhythmic deaths occurred among patients with VT/VF recurrence [5]. For example, analysis of the 6 deaths that occurred among the WCD group indicated that 5 occurred among those with immediately recurrent VT/VF. In contrast, 14 patients shocked for a single instance of life-threatening arrhythmia survived to the 90 days study endpoint [5]. As a result, patients at risk for SCD may in fact benefit from the WCD, although those experiencing arrhythmic storm are especially in need of timely access to advanced medical care. In the case of the WCD, physicians must continue to weigh the perceived risk of SCD against whether the absence of evidence reported by the VEST investigators is a valid enough justification for inaction $[14,15]$.

The European Society of Cardiology and the German Cardiology Association have published that the WCD can save lives in patients at risk for VT/VF [16, 17]. Current recommendations for WCD prescription are class IIa-c for use after an ICD explantation, diagnosis of acute myocarditis, and in patients on the heart transplantation waiting list $[16,17]$. It is recommended as class $\mathrm{IIb}-\mathrm{c}$ in a large number of patients with other cardiomyopathies who are expected to achieve improved LVEF (e.g., after coronary artery bypass graft, percutaneous coronary intervention, acute MI, dilated cardiomyopathy, and non-ischemic cardiomyopathy).

In Germany as well as other countries, the WCD is commonly prescribed to patients considered to be at risk for SCD and its efficacy is well documented. As a result, there is ample reason to believe that clinical equipoise will prevent the execution of new randomized control trials requiring that at risk patients are denied access to the WCD. It is common, however, to recommend multiple large-scale randomized control trials to define the treatment of therapy, particularly when reporting community-based studies. Based on our experience, we recommend that lives already saved by appropriate WCD shocks have provided sufficient support for classification of the WCD as a class I standard of care for the patient at risk for sudden cardiac arrest.

\subsection{Limitations}

The study has several strengths and limitations. This study is the first to describe a cohort of consecutive patients enrolled from a community-based acute care hospital serving a semirural region. Along with the PROLONG study cohort, this study is one of the first to report LVEF at the beginning and end of WCD use. Limitations include the absence of data on common cardiovascular comorbidities. The definitions and hierarchy used to assign patients to a single cardiac diagnosis likely resulted in overlap among disease etiologies. Additional limitations include the lack of a control group of patients who did not receive the WCD. The possibility that the use of a WCD leads to better care, and subsequent improvement in LVEF, may exist. Inherent to the study design of a nonrandomized observational analysis is the possibility of selection bias.

\section{Conclusions}

In a community care setting, the impact of WCD use on longterm decision for ICD implantation resulted from identifiable changes in clinical status, such as LVEF improvement or occurrence of VT/VF. Age, gender, and initial LVEF did not predict outcomes. During optimization of GDMT while using the WCD, patient clinical status can improve, resulting in a significantly decreased risk for SCD. Use of the WCD protects patients from SCD until response to GDMT is determined. 
Authors' contributions All authors have contributed significantly to the submitted work, drafting and revising critically the manuscript.

Funding Open Access funding enabled and organized by Projekt DEAL. The registry is supported by an unrestricted grant from ZOLL, Pittsburgh, PA, USA.

Data availability All patient data were collected, statistically analyzed, and archived in the Sana Klinikum Hof, Germany.

\section{Compliance with ethical standards}

Conflict of interest The authors declare that they have no conflict of interest.

Ethical approval Not applicable

Code availability Software application: R Core Team, R: A Language and Environment for Statistical Computing. R Foundation for Statistical Computing, Vienna, Austria. URL https://www.R-project.org/.

Open Access This article is licensed under a Creative Commons Attribution 4.0 International License, which permits use, sharing, adaptation, distribution and reproduction in any medium or format, as long as you give appropriate credit to the original author(s) and the source, provide a link to the Creative Commons licence, and indicate if changes were made. The images or other third party material in this article are included in the article's Creative Commons licence, unless indicated otherwise in a credit line to the material. If material is not included in the article's Creative Commons licence and your intended use is not permitted by statutory regulation or exceeds the permitted use, you will need to obtain permission directly from the copyright holder. To view a copy of this licence, visit http://creativecommons.org/licenses/by/4.0/.

\section{References}

1. Masterson S, Wright P, O'Donnell C, et al. Urban and rural differences in out-of-hospital cardiac arrest in Ireland. Resuscitation. 2015;91:42-7. https://doi.org/10.1016/j.resuscitation.2015.03.012.

2. Saner H, Morger C, Eser P, von Planta M. Dual dispatch early defibrillation in out-of-hospital cardiac arrest in a mixed urbanrural population. Resuscitation. 2013;84:1197-202. https://doi. org/10.1016/j.resuscitation.2013.02.023.

3. Larsen MP, Eisenberg MS, Cummins RO, Hallstrom AP. Predicting survival from out-of-hospital cardiac arrest: a graphic model. Ann Emerg Med. 1993;22:1652-8. https://doi.org/10. 1016/S0196-0644(05)81302-2.

4. Bardy GH, Lee KL, Mark DB, Poole JE, Toff WD, Tonkin AM, et al. Home use of automated external defibrillators for sudden cardiac arrest. N Engl J Med. 2008;358:1793-804. https://doi.org/ 10.1056/NEJMoa0801651.

5. Olgin JE, Pletcher MJ, Vittinghoff E, Wranicz J, Malik R, Morin DP, et al. Wearable cardioverter-defibrillator after myocardial infarction. N Engl J Med. 2018;379:1205-15. https://doi.org/10. 1056/NEJMoa1800781.
6. Wäßnig NK, Günther M, Quick S, Pfluecke C, Rottstädt F, Szymkiewicz SJ, et al. Experience with the wearable cardioverterdefibrillator in patients at high risk for sudden cardiac death. Circulation. 2016;134:635-43. https://doi.org/10.1161/ CIRCULATIONAHA.115.019124.

7. Kutyifa V, Moss AJ, Klein H, Biton Y, McNitt S, MacKecknie B, et al. Use of the wearable cardioverter defibrillator in high-risk cardiac patients: data from the prospective registry of patients using the wearable cardioverter defibrillator (WEARIT-II Registry). Circulation. 2015;132:1613-9. https://doi.org/10.1161/ CIRCULATIONAHA.115.015677.

8. Feldman AM, Klein H, Tchou P, Murali S, Hall WJ, Mancini D, et al. Use of a wearable defibrillator in terminating tachyarrhythmias in patients at high risk for sudden death: results of the WEARIT/BIROAD. Pacing Clin Electrophysiol. 2004;27:4-9. https://doi.org/10.1111/j.1540-8159.2004.00378.x.

9. Epstein AE, Abraham WT, Bianco NR, Kern KB, Mirro M, Rao $\mathrm{SV}$, et al. Wearable cardioverter-defibrillator use in patients perceived to be at high risk early post-myocardial infarction. J Am Coll Cardiol. 2013;62:2000-7. https://doi.org/10.1016/j.jacc.2013. 05.086.

10. Chung MK, Szymkiewicz SJ, Shao M, Zishiri E, Niebauer MJ, Lindsay BD, et al. Aggregate national experience with the wearable cardioverter-defibrillator: event rates, compliance, and survival. J Am Coll Cardiol. 2010;56:194-203. https://doi.org/10.1016/j.jacc. 2010.04.016.

11. R Core Team, R: A language and environment for statistical computing. R Foundation for Statistical Computing, Vienna, Austria. 2015. URL https://www.R-project.org/. Accessed 2015.

12. Duncker D, König T, Hohmann S, Bauersachs J, Veltmann C. Avoiding untimely implantable cardioverter/defibrillator implantation by intensified heart failure therapy optimization supported by the wearable cardioverter/defibrillator-the PROLONG study. J Am Heart Assoc. 2017;6. https://doi.org/10.1161/JAHA.116.004512.

13. Duncker D, König T, Hohmann S, Bauersachs J, Veltmann C. Ventricular arrhythmias in patients with newly diagnosed nonischemic cardiomyopathy: insights from the PROLONG study. Clin Cardiol. 2017;40:586-90. https://doi.org/10.1002/clc.22706.

14. Field ME, Page RL. Another shock for sudden death prevention after myocardial infarction. N Engl J Med. 2018;379:1274-5. https://doi.org/10.1056/NEJMe1809832.

15. Altman DG, Bland JM. Absence of evidence is not evidence of absence. BMJ. 1995;311:485. https://doi.org/10.1136/bmj.311. 7003.485 .

16. Priori SG, Blomström-Lundqvist C, Mazzanti A, et al. 2015 ESC guidelines for the management of patients with ventricular arrhythmias and the prevention of sudden cardiac death: the task force for the management of patients with ventricular arrhythmias and the prevention of sudden cardiac death of the European Society of Cardiology (ESC) endorsed by: Association for European Paediatric and Congenital Cardiology (AEPC). Europace. 2015;17:1601-87. https://doi.org/10.1093/europace/euv319.

17. Schwab JO, Bänsch D, Israel C, Nowak B. Opinion on the use of the portable cardioverter/defibrillator. Kardiologe. 2015;9:165-70.

Publisher's note Springer Nature remains neutral with regard to jurisdictional claims in published maps and institutional affiliations. 